\title{
Efeitos de intervenção educativa nos agentes comunitários de saúde sobre a caderneta de saúde da pessoa idosa de um município do Pará
}

\author{
Effects of educational intervention in community health agents on the health booklet of the \\ elderly in a municipality in Pará
}
Efectos de la intervención educativa en agentes comunitarios de salud en la cartilla de salud de los ancianos en un municipio de Pará

Jessyca Alves das Neves Costa ${ }^{1 *}$, Angélica Homobono Nobre ${ }^{1}$, Jorgeane Pedrosa Pantoja ${ }^{2}$.

\begin{abstract}
RESUMO
Objetivo: Avaliar os efeitos de intervenção educativa nos conhecimentos e práticas dos agentes comunitáriosde saúde sobre a caderneta de saúde da pessoa idosa de um município do Pará. Métodos: O estudo foi quase-experimental, do tipo antes e depois. O objeto de investigação foram os agentes comunitários que estavam em exercício da função. A coleta de dados ocorreu em três etapas,a primeira em formato online por preenchimento de questionário dividido em dados sociodemográficos, profissionais e sobre a caderneta, a segunda foi a intervenção com construção de diário de campo e após a intervenção com o preenchimento novamente do questionário online. Os dados obtidos foram analisados de forma quantitativa através do software BioStat 5.0 e de forma qualitativa pela análise de conteúdo de Bardin L (2021).O estudo foi aprovado por Comitê de Ética em Pesquisa. Resultados: A intervenção educativa mostrou-se eficaz na mudança dos conhecimentos e práticas dos agentes, principalmente referente as práticas. Conclusão: A melhora do conhecimento e práticas dos agentes revela a importância da educaçãopermanente no processo de trabalho de toda equipe. O estudo apresentou limitações principais a escassez de literaturas referentes a caderneta.
\end{abstract}

Palavras-chave: Saúde do idoso, Agentes comunitários de saúde, Educação permanente.

\begin{abstract}
Objective: To evaluate the effects of educational intervention on the knowledge and practices of community health agents about the health handbook for elderly of county of Pará. Methods: The study was quasi-experimental, of the before and after type. The target audience was community agents who were working. Data collection took place in three stages, the first in online format bEduy fillingout a questionnaire divided into sociodemographic, professional and booklet data, the second was the intervention with the construction of a field diary and after the intervention with the completion of the questionnaire again online. The data obtained were analyzed quantitatively using the BioStat 5.0 software andqualitatively using Bardin L (2021) content analysis. The study was approved by the Research Ethics Committee. Results: The educational intervention proved to be effective in improving the agents' knowledge and practices,especially regarding conduct. Conclusion: The improvement of agents knowledge and practices reveals the importance of continuing education in the entire team's work process. The main limitations of the present studywere the scarcity of literature referring to the booklet.
\end{abstract}

Keywords: Health of the elderly, Community health workers, Education continuing.

\section{RESUMEN}

Objetivo: Evaluar los efectos de la intervención educativa sobre los conocimientos y prácticas de los agentes comunitarios de salud sobre el cartilla de salud del adulto mayor en un municipio de Pará. Métodos:El estudio fue cuasiexperimental, del tipo antes y después. El público objetivo fueron los agentes comunitarios que se encontraban trabajando. La recogida de datos se llevó a cabo en tres etapas, la primera en formato online mediante la cumplimentación de un cuestionario dividido en datos sociodemográficos, profesionales y cuadernillo, la segunda fue la intervención con la construcción de un diariode campo y posterior a la intervención con la cumplimentación del cuestionario nuevamente. en línea. Los datos obtenidos se analizaron cuantitativamente utilizando el software BioStat 5.0 y cualitativamente utilizandoel análisis de contenido de Bardin L (2021). El estudio fue aprobado por el Comité de Ética en Investigación. Resultados: La intervención educativa demostró ser efectiva para mejorar los conocimientos y prácticas de los agentes, especialmente en lo referente a la conducta. Conclusión: La mejora de los conocimientos y prácticas de los agentes revela la importancia de la educación continua en el proceso de trabajo de todo el equipo. Las principales limitaciones del presente estudio fueron la escasez de literatura referente al folleto.

Palabras clave: Salud del anciano, Agentes comunitarios de salud, Educación continua.

${ }^{1}$ Universidade do Estado do Pará, Belém - PA. *E-mail: fisiojessyca@gmail.com

2 Universidade Federal do Pará, Belém - PA. 


\section{INTRODUÇÃo}

As alterações decorrentes do envelhecimento ocorrem em diversos aspectos como social, de saúde e previdenciário. O aumento da demanda por serviços de saúde, dos índices de morbidade e das doenças crônicodegenerativas são qualificadas como questões de saúde pública. Nesse âmbito, os idosos são a parcela da população que mais utiliza o Sistema Único de Saúde (SUS), demandando maiores custose adaptações para uma assistência eficaz (COSTA NRCD, et al., 2016).

Dentre as estratégias para acompanhamento da população idosa, a Caderneta de Saúde da Pessoa Idosa (CSPI) foi lançada a nível nacional em 2007 e atualmente está em sua 5a edição (2018). Em determinação do Ministério da Saúde (MS), o setor de atenção primária foi o primeiro a receber o instrumento. Nesse nível de atenção, a caderneta é disponibilizada pela Estratégia de Saúde da Família (ESF) como forma de fortalecer o vínculo e zelar pelo estado de saúde desses indivíduos (MINISTÉRIO DA SAÚDE, 2017).

A equipe de estratégia saúde da família com sua composição multiprofissional tem no Agente Comunitário de Saúde (ACS) o multiplicador em saúde entre os usuários, pois ele se constitui como primeiro e principal contato com a Unidade Básica de Saúde (UBS). Esse profissional deve ser constantemente capacitado, principalmente no que compete aos instrumentos utilizados para qualificar a atenção primária, como é o caso da Caderneta de Saúde da Pessoa Idosa (MINISTÉRIO DA SAÚDE, 2017).

$\mathrm{O}$ insturmento em questão integra um conjunto de iniciativas de reconhecimento de vulnerabilidades e manejo do cuidado e pode ser usado pelos profissionais de saúde, pelos idosos e por seus familiares e cuidadores. No entanto para o correto uso dessa caderneta são necessárias ações educativas capazes de fomentar discussões e impactar a prática através da educação em saúde (ALBUQUERQUE MRTC, et al., 2020).

Nesse contexto, a qualificação desses profissionais é um ato estratégico e de fortalecimento da díade usuário-unidade, portanto o objetivo deste estudo foi avaliar os efeitos de intervenção educativa nos conhecimentos e práticas dos agentes comunitários de saúde sobre a caderneta de saúde da pessoa idosa.

\section{MÉTODOS}

Trata-se de estudo quase-experimental, não randomizado, baseado em pré e pós intervenção. Foram analisadas as mudanças relativas aos conhecimentos e práticas sobre o uso da caderneta de saúde da pessoa idosa ocorridas no processo de trabalho dos agentes comunitários de saúde antes e após uma intervenção educativa. A pesquisa desenvolveu-se em um município do estado do Pará. O município possui uma área de 187,826 km², uma população estimada de 63.768 habitantes e $100 \%$ de cobertura pela ESF (MINISTÉRIO DA SAÚDE, 2020).

A amostra foi definida de forma não probabilística, mediante critérios de inclusão/exclusão. Constituíram os critérios de inclusão da pesquisa estar atuando como ACS no período do estudo e possuir no mínimo umano de experiência no cargo. Em relação ao critério de exclusão, foram desconsiderados agentes que estivessem de férias ou licença, que participaram de roda de conversa semelhante ao assunto do estudo realizada no ano anterior pela pesquisadora e os que não participaram de todas as etapas do estudo. Houveuma perda de 22 participantes pelos seguintes motivos: não quiseram participar (3); férias/licença (11); não preencheram o pósteste (8). A população do estudo foi composta por 98 agentes. A coleta de dados ocorreuno período de maio a setembro de 2021.

A coleta de dados foi dividida em três momentos: a primeira etapa foi o preenchimento do questionário online, a segunda etapa foram as intervenções educativas presenciais baseadas na educação popular em saúde com presença de rodas de conversa, os ACS foram divididos em quatro grupos, cada grupo realizou aintervenção durante uma hora e 30 minutos em dias e horários separados; a terceira etapa foi o preenchimento, após 30 dias da intervenção educativa, do mesmo questionário online porém com o adendo de duas questões abertas relacionadas às dificuldades e condutas dos ACS. O uso dos questionários onlinefoi preconizado devido as questões relativas à pandemia de COVID-19 e a distância e dificuldade de acessoas unidades de saúde do município, optando dessa forma pelo uso da tecnologia em questão.

Os instrumentos utilizados para a coleta foram: 1) os questionários produzidos pela pesquisadora via formulário online, o primeiro (pré-teste) contava com duas sessões - sociodemográfica/profissionais e conhecimentos e práticas e o segundo (pós-teste) com as mesmas questões do primeiro acrescido de duas 
questões abertas: I) Quais as principais dificuldades encontradas por você para aplicar na prática os conhecimentos adquiridos na oficina? e II) Após a intervenção, quais condutas você adota frente ao paciente idoso no dia a dia de visitas e acompanhamentos?. Além dos questionários, a pesquisadora produziu um diário de campo com falas dos participantes durante a intervenção.

Para análise e organização dos dados quantitativos, utilizou-se o Software Bioestat versão 5.0. As variáveis contínuas foram expressas por média e desvio-padrão (DP) e as categóricas, por frequências absolutas e relativas. Para comparar as diferenças nos percentuais de acertos nos períodos pré e pós- intervenção, utilizouse o teste de McNemar. Considerou-se o nível de significância de $p$-valor $(p<0,05)$.

O diário de campo produzido durante a intervenção educativa e o questionário aplicado após a intervençãocom as perguntas abertas foram analisados conjuntamente, seguindo a técnica de análise de conteúdo, pela modalidade de temática transversal. (BARDIN L, 2011). Para a composição do corpus da análise qualitativa foi observada a saturação teórica das respostas e as falas componentes do diário de campo.

O desenvolvimento deste estudo respeitou os preceitos éticos de pesquisa com seres humanos, estandode acordo com a Resolução no 466/12 do Conselho Nacional de Saúde, foi aprovado pelo Comitê de Ética e Pesquisa da Universidade do Estado do Pará, conforme parecer no 4.636.291/2021, CAAE no 44669521.2.0000.5174 e pela secretaria de saúde do município. Os participantes assinalaram o Termo de Consentimento Livre e Esclarecido (TCLE) de modo online comprovando a anuência em participar do estudo e para manutenção da confidencialidade dos participantes as falas são apresentadas em forma de código.

\section{RESULTADOS E DISCUSSÃO}

Os participantes da pesquisa apresentaram a idade média de 39,12 anos (Mínima:25; Máxima:63; DP:8,86), $82,6 \%$ mulheres e $17,3 \%$ homens. Observou-se que a maioria, 70,4\% tinham o ensino médio completo e 14,2\% possuíam o ensino superior completo. A maioria, 52\% possuía como tipo de vínculo o concurso e a $75,51 \%$ possuíam até 12 anos de atuação (Tabela 1).

Tabela 1 - Distribuição dos Profissionais segundo as variáveis sociodemográficase profissionais $n=98$.

\begin{tabular}{lcc}
\hline Variável & N & $\%$ \\
\hline Gênero & & \\
\hline Feminino & 81 & 82,65 \\
Masculino & 17 & \\
\hline Escolaridade & 69 & 70,41 \\
\hline Médio Completo & 12 & 12,24 \\
Superior Incompleto & 14 & 14,29 \\
Superior Completo & 1 & 1,02 \\
Pós-graduação incompleta & 2 & 2,04 \\
Pós-graduação completa & & \\
\hline Tipo de Vínculo & 51 & 52,04 \\
\hline Concurso & 16 & 16,33 \\
Contrato/CLT & 5 & 5,10 \\
Estatutário & 26 & 26,53 \\
Processo Seletivo & & \\
\hline Tempo de Atuação & 74 & 75,51 \\
\hline Até 12 anos & 24 & 24,48 \\
12 anos e mais & & \\
\hline
\end{tabular}

Fonte: Costa JAN, et al., 2022.

As informações sociodemográficas e profissionais dos participantes do estudo demonstraram semelhanças aos de outro quanto serem do gênero feminino, possuírem o ensino médio completo e uma média de tempo de serviço maior que 5 anos (PAIVA KM, et al., 2019). Este resultado pode estar ligado ao papel que a mulher desempenha na sociedade como cuidadora, tanto na vida das crianças quanto na prestação de cuidados aos idosos de seus núcleos familiares (FERREIRA CR, et al., 2018).

A caderneta contém informações fundamentais como: Dados pessoais do idoso, medicamentos, protocolode identificação do idoso vulnerável, avaliação do ambiente domiciliar, risco de quedas, mapeamento da dor crônica, monitoramento de doenças crônicas como hipertensão e diabetes, calendário vacinal e outras demandas (MINISTÉRIO DA SAÚDE, 2018a). 
Cerca de 4060 idosos são acompanhados pela estratégia saúde da família do município, logo as informações da caderneta auxiliam a equipe de saúde a desenvolver um plano de cuidados que atenda de forma qualificada às necessidades da população idosa, portanto, é muito importante que os profissionais conheçam e utilizem o instrumento (BRITO TEDS, et al., 2019).

$\mathrm{Na}$ Tabela 2, são apresentadas as questões referentes aos conhecimentos relacionados à caderneta de saúde da pessoa idosa e a porcentagem de acertos antes e depois da intervenção educativa. Observa-se quea maioria dos itens obtiveram um aumento na taxa de acertos nos pós teste.

Tabela 2 - Distribuição dos resultados referentes ao conhecimento sobre acaderneta.

\begin{tabular}{lccc}
\hline Conhecimentos & Antes(\%) & Depois(\%) & $\mathbf{p}^{*}$ \\
\hline $\begin{array}{l}\text { Quando você identifica que o idoso mora sozinho, esse fato indica } \\
\text { uma situação de vulnerabilidade? }\end{array}$ & 89,79 & 93,88 & 0,4533 \\
\hline $\begin{array}{l}\text { Pessoas idosas que utilizam cinco ou mais tipos de medicamentos } \\
\text { são consideradas de risco? }\end{array}$ & 91,84 & 96,94 & 0,2278 \\
\hline $\begin{array}{l}\text { A perda de peso não intencional é um fatorimportante na fragilidade } \\
\text { do idoso? }\end{array}$ & 100,00 & 97,96 & 0,4795 \\
\hline $\begin{array}{l}\text { ldosos que residem em instituições de longa permanência não } \\
\text { precisam da caderneta de saúde da pessoa idosa? }\end{array}$ & 45,92 & 51,02 & 0,5677 \\
\hline Objetivo da caderneta de saúde é: & 82,65 & 88,78 & 0,2864 \\
\hline $\begin{array}{l}\text { Utilizando a caderneta de saúde da pessoaidosa é possível } \\
\text { identificar um idoso vulnerável no seu território? }\end{array}$ & 93,88 & 97,96 & 0,2891 \\
\hline
\end{tabular}

Fonte: Costa JAN, et al., 2022.

Referente ao conhecimento é possível inferir que os agentes possuíam o entendimento sobre os tópicos da caderneta e após a intervenção foi observado o aumento da porcentagem de acertos das perguntas.

Estudos têm demonstrado que os profissionais reconhecem a CSPI ao conceituar e dar sentido ao instrumento, como uma ferramenta para melhora do processo de trabalho, da comunicação entre os profissionais da APS e os idosos e para fornecer e divulgar informações sobre a saúde do idoso.

(CARVALHÊDO FG, et al., 2015; SILVA KM e SANTOS SMA, 2015; SÁ CMP, 2016).

Um documento que é gratuitamente disponibilizado, de linguagem sucinta e ilustrado que possuicomo cerne aproximar o cuidado à saúde da população, influenciando o protagonismo e autonomia, mais do que conhecer os conteúdos presentes na caderneta a importância deste instrumento perpassa pelo desenvolvimento de competências à saúde tanto para os profissionais quanto para os usuários e seus familiares (SILVA TN e CHACON PF, 2020).

Quanto às questões referentes às práticas relacionadas a caderneta, a Tabela 3 apresenta a distribuição da porcentagem de acertos pré e pós intervenção.

Tabela 3 - Distribuição dos resultados referentes as práticas relacionadas acaderneta.

\begin{tabular}{lccc}
\hline Práticas & Antes (\%) & Depois (\%) & $\mathbf{p}^{*}$ \\
\hline $\begin{array}{l}\text { Durante a realização de uma visita domiciliar, você solicita a } \\
\text { caderneta de saúde da pessoa idosa? }\end{array}$ & 97,96 & 98,98 & 1 \\
\hline $\begin{array}{l}\text { Você observa as condições do ambientedomiciliar quando realiza } \\
\text { uma visita? }\end{array}$ & 100,0 & 100,00 & 1 \\
\hline $\begin{array}{l}\text { Você explica para a pessoa idosa efamiliares como usar a } \\
\text { caderneta? }\end{array}$ & 97,96 & 100,00 & 0,5 \\
\hline $\begin{array}{l}\text { Você costuma identificar um idoso vulnerável no seu território } \\
\text { utilizando a caderneta? }\end{array}$ & 91,84 & 95,92 & 0,34 \\
\hline $\begin{array}{l}\text { Você realiza as principais orientações sobrealimentação, direitos } \\
\text { da pessoa idosa,prática de atividade física e saúde bucal? }\end{array}$ & 98,98 & 100,00 & 1 \\
\hline $\begin{array}{l}\text { Quando uma pessoa idosa comunica quecaiu você registra na } \\
\text { caderneta? }\end{array}$ & 79,59 & 81,63 & 0,85 \\
\hline $\begin{array}{l}\text { Na falta da caderneta de saúde da pessoa idosa, você utiliza a } \\
\text { ficha-espelho (ficha quecontém as informaçóes da caderneta e } \\
\text { que é disponibilizada pelo Ministério da Saúde)? }\end{array}$ & 41,84 & 71,43 & $<0,0001$ \\
\hline $\begin{array}{l}\text { Você verifica se as vacinas do idoso se encontram atualizadas? } \\
\text { E caso não estejamvocê orienta para atualizar? }\end{array}$ & 100,00 & 100,00 & 1 \\
\hline
\end{tabular}

Fonte: Costa JAN, et al., 2022. 
Após a intervenção educativa, a prática referente ao uso da ficha espelho na ausência da caderneta apresentou associação significativa, com aumento de $29,59 \%$ na utilização pelos ACSs. Em relação à práticade análise ambiental, $100 \%$ dos participantes relataram já realizar a observação do ambiente familiar, antes e depois da intervenção.

No pré-teste os ACS apresentavam pouco envolvimento com práticas relacionadas ao uso da ficha- espelho, resultado esse semelhante ao observado em outro estudo de Schmidt A, et al. (2019), no qual as pesquisadoras destacam o desconhecimento do uso prático da caderneta e seus anexos pelos membros da equipe os quais relacionam a caderneta como uma ferramenta estática e a distanciando do cuidado continuado e da prática diária.

Após a análise dos dados qualitativos através da exploração do material emergiram duas categorias temáticas: Categoria 1 - $O$ impacto do instrumento de trabalho e da educação permanente na rotina da equipe e Categoria 2 - O efeito da intervenção na prática do ACS sobre a saúde da população idosa.

\section{Categoria 1 - 0 impacto do instrumento de trabalho e da educação permanente na rotina da equipe.}

Essa categoria emergiu das falas dos participantes durante a intervenção e das respostas relacionadas às principais dificuldades no acompanhamento da pessoa idosa na APS. Os ACS abordaram como fator principala escassez do material em formato físico, das capacitações multiprofissionais e como isso influencia negativamente na conduta de toda equipe, assim como o entendimento dos usuários e demais profissionais sobre a relevância do instrumento em questão.

\section{"A falta de caderneta no município tenho 66 idosos porém somente 45 tem caderneta" (ACS1). \\ "Falta de materiais tipo a fita métrica, aparelho de PA e a caderneta" (ACS2). \\ "Não possuímos a caderneta para dar aos idosos, é muito difícil trabalhar sem os materiais que precisamos. Os idosos chegam no posto e só nós sabemos quase tudo sobre cada um, tudo bem que é o nosso papel como ACS, mas os outros profissionais também tem que saber mais sobre os pacientes deles[...]" (ACS2).}

Observam-se duas situações. A primeira é a ausência de cadernetas suficientes no município, que são distribuídas pelo Ministério da Saúde (MS), de acordo com as necessidades indicadas por cada entidade municipal. A segunda, a pouca utilização das cadernetas de idosos pelos profissionais (daqueles idosos que possuem). Como exemplifica a seguinte fala:

"A falta da carteira, dificulta muito nosso trabalho, e os profissionais que atendem o nosso idoso que muitasdas vezes atendem este idoso e não colocam nada na carteira" (ACS3).

Para além da falta do instrumento, a baixa adesão do material relatada pelos ACS por parte dos demais profissionais fortalece a crença dos idosos de que a caderneta não precisa ser utilizada com frequência prejudicando o acompanhamento e o cuidado integral desses usuários. Algumas lacunas podem dimensionaro porquê do pouco uso da caderneta e são apontadas por Schmidt A, et al.(2019) tais como o desconhecimento do uso prático, carga de trabalho excessiva e pouco conhecimento técnico científico de gerontologia entre os profissionais de saúde.

Esse achado também foi relatado por outro estudo, no qual os agentes citaram os principais entraves na implementação de uma rede de atenção eficiente para as pessoas idosas, tais como a dificuldade de alinharos pensamentos da equipe e o déficit de recurso (CABRAL JF, et al.,2019).

Como equipamentos básicos para a execução do trabalho do ACS o ministério da saúde fornece: a vestimenta, identificação, material educativo e outros. No estudo de Costa NRCD, et al., (2016) o não recebimento das cadernetas e a distribuição sem a qualificação profissional adequada foram apontados comoos pontos negativos da política nacional da pessoa idosa.

"A grande dificuldade é que não temos caderneta do idoso aí estas questões são inviáveis” (ACS4). 
Um fato impeditivo na resolutividade e alcance da população nas unidades básicas é a escassez de insumos e equipamentos, tal fator limita a capacidade de resposta aos problemas de saúde dos territórios (CABRAL JF, et al., 2019). Outro ponto crucial de impedimento na resolutividade da atenção é a baixa qualificação profissional ressaltada na fala dos participantes que está diretamente interligada ao processo deeducação permanente em saúde (EPS).

Os agentes comunitários pontuaram que a necessidade da educação permanente em saúde deve ser estendida aos demais membros da equipe e que muitas vezes o desconhecimento dos demais, pode influenciar no processo de trabalho dos agentes, assim como nas decisões do uso ou não-uso da cadernetapela população idosa.

"Os poucos idosos que tem a caderneta, não levam pro posto porque dizem que 0 médico e a enfermeiranão a usam, logo eles não têm porque levar pra consulta. O que não deixa de ser verdade, porque é realmenteignorado durante as consultas. Quando eu vou visitar eu nem peço pra olhar porque eu sei que ela vai estar em branco [...]" (ACSD4).

"[...] é muito importante momentos como esse, mas o mais importante é que ele aconteça com toda a equipe. Muitos profissionais não utilizam a carteira, o idoso vai lá na unidade e recebe papeiszinhos pra anotara pressão e fica sem usar a carteira [...]" (ACSA4).

A EPS caracteriza-se por um processo educativo que enfoca as necessidades e problemáticas coletivas identificadas no espaço micropolítico do trabalho em saúde e promove a transformação de aprendizados e práticas necessárias para o alcance do cuidado integral. Portanto, a prática da EPS está diretamente relacionada à forma como a equipe organiza o processo de trabalho e aquilo que o embasa (DOLNY LL, et al., 2020).

A transformação e desenvolvimento das ações voltadas à saúde do idoso perpassam pela educação permanente em saúde de modo que os profissionais se sintam envolvidos e orientados, além de incentivadosà atender de forma resolutiva e eficaz as necessidades do público idoso (RAMOS LV, 2019). Uma ação educativa possui maior significado quando ocorre de forma não verticalizada, ou seja, pela transmissão unilateral de conhecimentos (RAMOS LV, et al.,2019). Nesse sentido, a troca de experiências e o compartilhamento dos conhecimentos entre os profissionais foram apontados por estes como algo significativo durante 0 desenvolvimento dessa intervenção, refletindo sobretudo na prática dos ACS.

Outro estudo observou que nenhum dos profissionais indicou em sua atuação o preenchimento da CSPI, ainda que ela seja preconizada para o acompanhamento longitudinal da pessoa idosa, essa atitude também é exemplificada nas falas dos participantes (SÁ CMP, 2016). As ações voltadas para a população idosa tornam-se prejudicadas sem o correto preenchimento e uso da CSPI.

\section{Categoria 2 - $O$ efeito da intervenção na prática do ACS sobre a saúde da população idosa.}

Nesta categoria, destacam-se mudanças implementadas na prática da atuação do ACS após a intervenção. Nas respostas às perguntas abertas foram apresentadas mudanças em pontos como o entendimento e priorização da identificação do idoso vulnerável na rotina da visita domiciliar e no acompanhamento quanto aos aspectos específicos da pessoa idosa. Os ACS apresentaram maior sensibilidade quanto ao uso da caderneta, priorizando as informações de orientação familiar, atitudes que refletiram nas condutas diárias dos profissionais.

"[...]Eu não sabia que dava pra saber se o idoso é frágil ou não através da carteira dele. Na maioria dasvezes via apenas a vacina, mas agora vou prestar mais atenção [...]" (ACSB1).

"[...]A partir dessa capacitação meus conhecimentos foram ampliados e me sinto mais segura em repassaras informações que poderão servir de maior uso para a prevenção de doenças como no caso de diabetes a cada consulta, anotação da glicemia e estar sempre acompanhando [...]" (ACSB4).

Em estudo de Seibert G, et al. (2020), os ACS após capacitação demonstraram a adoção de estratégias mais alinhadas as necessidades do território e norteadas pelos tópicos contidos na caderneta. $O$ crescente aumento 
da população idosa torna imprescindível a adoção de estratégias e táticas voltadas para esse grupo,com vistas a otimizar a autonomia, participação e um envelhecimento seguro, dessa forma o uso da ficha- espelho torna-se um ponto eficaz no acompanhamento da pessoa idosa.

Segundo dados do Ministério da Saúde cerca de 3.035 municípios brasileiros aderiram à Caderneta de Saúde da Pessoa Idosa, a orientação fornecida pelo MS é que as equipes da APS as quais os municípios ainda não possuem a Caderneta devem utilizar a ficha espelho disponibilizada em endereço eletrônico (MINISTÉRIO DA SAÚDE, 2018b).

Os participantes relataram utilizar em sua conduta a ficha-espelho após o momento educacional promovidopela pesquisadora.

"Oriento melhor os idosos da minha área, consigo usar a ficha espelho, tenho mais diálogo com osfamiliares principalmente sobre quedas e outros riscos [...]" (ACSD2).

"O uso da ficha espelho no prontuário, orientações para as atividades de vida e qualquer registroimportante deve ser feito na ficha." (ACS1).

"Oriento quanto aos riscos de cair e quanto a melhora de hábitos porque hábitos ruins podem levar afragilidade [...]" (ACS2).

O uso da ficha-espelho da caderneta contribui para monitorar as condições de saúde e a atenção fornecidaà pessoa idosa, favorecendo o planejamento e organização de ações dos ACS e da equipe como um todo. Possuir esse registro da ficha-espelho no prontuário torna-se um estímulo para o uso da caderneta em si.

Em concordância com as respostas dos ACS, no estudo de Anjos KF, et al. (2020) foram observadas mudanças nas responsabilidades dos agentes após educação em saúde, principalmente no que compete as práticas das visitas domiciliares. Atividades educativas modificam não apenas o processo de trabalho do ACSmas a compreensão e aceitação ao idoso refletindo em condutas pautadas na maior independência da pessoaidosa (NICODEMOS FURTADO DE MENDONÇA FT, et al., 2017).

\section{CONCLUSÃO}

No presente estudo foi observado uma repercussão positiva no conhecimento e melhora nas práticas dos agentes comunitários de saúde no que concerne ao instrumento da caderneta de saúde da pessoa idosa, principalmente sobre o uso da ficha-espelho. Sugere-se a realização de mais estudos que explorem mais amplamente a caderneta e a saúde do idoso. Ademais, este estudo pontuou que a necessidade de investir na educação permanente tanto dos ACS quanto de toda a equipe da ESF é um pronto estratégico para o desenvolvimento integral da saúde do idoso, tal ação é capaz de alavancar o manejo da CSPI e influenciar positivamente o processo de trabalho das equipes e o cuidado em saúde da população idosa no município.

\section{REFERÊNCIAS}

1. AlBUQUERQUE MRTC, et al. Caderneta de Saúde da Pessoa Idosa: Qualificando Agentes Comunitários de Saúde. Brazilian Journal of Health Review, 2020; 3(5): 13315-13324.

2. ANJOS KF, et al. Influencia de prácticas educativas de salud sobre responsabilidades deenfermeras, agentes de salud y cuidadores familiares para el cuidado de ancianos dependientes. Revista Cubana de Enfermería, 2020; 36(4).

3. BARDIN L. Análise de conteúdo. 1aㅡ. ed. São Paulo: Editora Edições 70, 2011; 209 p.

4. BRITO TEDS, et al. Utilização da caderneta de saúde da pessoa idosa por profissionais da saúde.Anais VI CIEH.Campina Grande: Realize Editora, 2019.

5. CABRAL JF, et al. Avaliação da atenção integral à saúde do idoso na percepção deprofissionais. Revista brasileira em promoção da saúde, 2019, 32.

6. CARVALHÊDO FG, et al. Acolhimento ao idoso e sistematização da assistência de enfermagem naatenção primária. Rev. enferm. UFPE online, 2015; 9(1): 143-148.

7. COSTA NRCD, et al. Política de saúde do idoso: percepção dos profissionais sobre suaimplementação na Atenção Básica. Revista de Pesquisa em Saúde, 2016; 16(2).

8. DOLNY LL, et al. Educação permanente em saúde (EPS) no processo de trabalho de equipes desaúde da família (ESF). Brazilian Journal of Health Review, 2020; 3(1): 15-38. 
9. FERREIRA CR, et al. Cuidar de idosos: um assunto de mulher?. Est. Inter. Psicol.2018; 9(1): 108-125.

10. MINISTÉRIO DA SAÚDE. Secretaria de Atenção Primária à Saúde. Departamento de Saúde da Família. Cobertura de Atenção Básica no período de julho de 2020 do município de Benevides-PA. 2020. Disponível em: https://egestorab.saude.gov.br/paginas/acessoPublico/relatorios/rellHistoricoCoberturaAB.xtml. Acessado em: 4 de dez. de 2021.

11. MINISTÉRIO DA SAÚDE. Secretaria de Atenção à Saúde. Departamento de Ações Programáticas Estratégicas. Caderneta de saúde da pessoa idosa. 2018a.Disponivel em: https://bvsms.saude.gov.br/bvs/publicacoes/caderneta_saude_pessoa_idosa_5ed.pdf. Acessado em: 4 de dez. de 2021.

12. MINISTÉRIO DA SAÚDE. Orientações Técnicas para Implementação de Linha de Cuidado para Atenção Integral à Saúde da Pessoa Idosa no Sistema Único de Saúde. 2018b. Disponível em: https://bvsms.saude.gov.br/bvs/publicacoes/linha_cuidado_atencao_pessoa_idosa.pdf. Acessado em: 4 de dez. de 2021.

13. MINISTÉRIO DA SAÚDE. Portal da Saúde. Caderneta de saúde da pessoa idosa. Brasília:Ministério da Saúde. 2017. Disponível em: https://portalarquivos2.saude.gov.br/images/pdf/2017/setembro/27/CADERNETA-PESSOA-IDOSA2017-Capamiolo.pdf. Acessado em: 04 dez. de 2021.

14. PAIVA KM, et al. Atenção ao idoso: percepções e práticas dos Agentes Comunitários de Saúde emuma capital do sul do Brasil. Sociedade Brasileira de Fonoaudiologia, 2019;31.

15. RAMOS LV, et al. Caderneta de saúde da pessoa idosa na atenção primária: uma revisãointegrativa. Humanidades \& Inovação,2019;6(2):272-280.

16. RAMOS LV. A utilização da caderneta de saúde da pessoa idosa na atenção primária: um olhar na formação em saúde. Dissertação (Mestrado em Ensino em Ciência e Saúde). Universidade Federal do Tocantins, Palmas, $2019 ; 108$ p.

17. SÁ CMCP. Caderneta de Saúde da Pessoa Idosa no Olhar dos Profissionais da Estratégia de Saúde da Família.Dissertação (Programa de Pós Graduação em Enfermagem) - Centro de Ciências da Saúde. Universidade Federal da Paraíba, João Pessoa, 2016; 74 p.

18. SCHMIDT A, et al. Preenchimento da caderneta de saúde da pessoa idosa: relato de experiência. SANARE-Revista de Políticas Públicas, 2019; 18(1).

19. SEIBERT G, et al. Relato de experiência Implantação da caderneta de saúde dapessoa idosa. Salão do Conhecimento, 2020; 6(6).

20. SILVA KM, SANTOS SMA. A práxis do enfermeiro da ESF e o cuidado ao idoso. Texto ContextoEnfermagem, 2015; 24(1): 105-11.

21. SILVA TN, CHACON PF.Caderneta de saúde da pessoa idosa como ferramenta de literacia paraa saúde. Revista Família, Ciclos de Vida e Saúde no Contexto Social, 2020; 3: 1064-1070. 